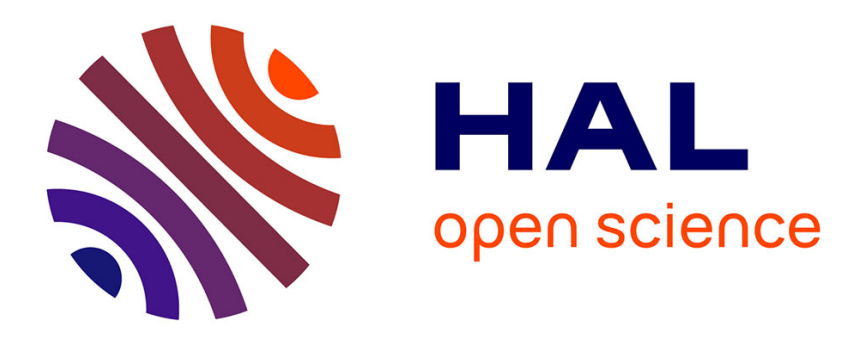

\title{
HYDRODYNAMIC EQUATIONS FOR NUCLEAR MATTER
}

\author{
W. Nawrocka
}

\section{To cite this version:}

W. Nawrocka. HYDRODYNAMIC EQUATIONS FOR NUCLEAR MATTER. Journal de Physique Colloques, 1984, 45 (C6), pp.C6-137-C6-142. 10.1051/jphyscol:1984616 . jpa-00224217

\section{HAL Id: jpa-00224217 https://hal.science/jpa-00224217}

Submitted on 1 Jan 1984

HAL is a multi-disciplinary open access archive for the deposit and dissemination of scientific research documents, whether they are published or not. The documents may come from teaching and research institutions in France or abroad, or from public or private research centers.
L'archive ouverte pluridisciplinaire HAL, est destinée au dépôt et à la diffusion de documents scientifiques de niveau recherche, publiés ou non, émanant des établissements d'enseignement et de recherche français ou étrangers, des laboratoires publics ou privés. 
JOURNAL DE PHYSIQUE

Colloque C6, supplément au $n^{\circ} 6$, Tome 45, juin 1984

page $\mathrm{C} 6-137$

HYDRODYNAMIC EQUATIONS FOR NUCLEAR MATTER

W. Nawrocka

Institute of Theoretical Physics, University of Wrockaw, Wroctow, Pozand

Résumé - Le comportement de la matière nuclëaire pénétrẻe par un nuclẻon est étudiée en approximation hydrodynamique.

Abstract - The behaviour of nuclear matter penetrated by a nucleon is considered in the hydrodynamic approximation.

1. For the nuclear processes with the large energy and momentum transfer while. the great number degrees of freedom is excited the statistical aspects are dominant. At the same time different collective variables are observed and measured. With this connection semi-classical hydrodynamic- like theoretical methods are regarded [1]. In such methods the only concerns are the time development of averaged quantities like density distribution, current density, thermal energy density etc, from which another collective observables can be computed.

At low energy excitations $\left(\frac{E_{C}}{A} \lesssim 10 \mathrm{MeV}\right)$ time dependent mean field approximation seems to be well applicable and two body collisions between quasiparticles can be assumed negligible [2]. At very high excitation energy in relativistic region the hydrodynamic picture with predominant two-body collisions is presumab1y valid [3]. From theoretical point of view intermediate between this two extremes is a more difficult energy region [4].

In this paper we consider the following simple model. One particle with mass $M$ penetrate the nuclear matter. We obtain the hydrodynamic equations describing the collective excitations of system at this process.

2. The nuclear matter one-particle distribution function is $f \equiv f(\vec{x}, \vec{p}, t)$. Penetrating particle distribution function we put for simplicity 


$$
g(\vec{x}, \vec{p}, t)=\delta\left(\vec{p}-\vec{p}_{0}\right) \delta(\vec{x}-\vec{u} t)
$$

where $\vec{u}=\frac{\vec{p}}{M}$. Kinetic equation for the $f$ can be written as

$$
\frac{\partial f}{\partial t}+\vec{v} \frac{\partial f}{\partial \vec{x}}=I_{1}(f, f)+I_{2}(f, g)
$$

where $I_{1}$ is the Boltzmann collision integral for particles of the medium, $I_{2}$ describes collisions of the penetrating particle with the particles of the medium. At the assumption (1)

$$
I_{2}(f, g)=\delta(\vec{x}-\vec{u} t) \int C\left(f \mid \vec{p}, \overrightarrow{p_{o}}, \vec{p}+, \vec{p} \mu\right) d^{3} p^{\prime} d^{3} p^{\mu}
$$

where

$$
\begin{aligned}
& C\left(f \mid \vec{p}, \vec{p}_{0}, \vec{p}^{-}, \vec{p}^{-\infty}\right)=\frac{1}{\pi}\left[\frac{d \sigma\left(s^{\prime}\right)}{d t} \cdot v_{r e 1}^{-2} f^{-} \delta^{(4)}\left(p^{-}+p_{\circ}-p-p^{-\infty}\right)+\right. \\
& \left.-\frac{d \sigma(s)}{d t} \cdot v_{r e 1}^{2} f \delta^{(4)}\left(p+p_{o}-p^{-}-p^{-\prime}\right)\right], \\
& f^{\prime} \equiv f\left(\vec{x}, \vec{p}^{-}, t\right), \quad v_{r e 1}^{\prime}=\left|\vec{v}^{-}-\vec{u}\right|, \quad v_{r e 1}=|\vec{v}-\vec{u}|, \\
& s, s^{-}-\text {the square of the total energy of colliding particles, } \\
& t \text { - the square of the momentum transfer from } \vec{p} \text { to } \vec{p}^{-} \text {. }
\end{aligned}
$$

To obtain the hydrodynamic equations let us consider the expressions

$$
\begin{aligned}
& \int I_{2}(f, g) d^{3} p, \\
& \int \vec{p} I_{2}(f, g) d^{3} p, \\
& \text { and } \quad \int \varepsilon I_{s}(f, g) d^{3} p
\end{aligned}
$$


where $\quad \varepsilon=\frac{\vec{p}^{2}}{2 \mathrm{~m}}$.

The integrals $(5 \mathrm{~b}),(5 \mathrm{c})$ are not equal zero because collisions $\left(\overrightarrow{\mathrm{p}}, \overrightarrow{\mathrm{p}}_{0}\right) \rightarrow(\overrightarrow{\mathrm{p}}, \overrightarrow{\mathrm{p}}$ ”) and $\left(\vec{p}^{-}, \vec{p}_{0}\right) \rightarrow\left(\vec{p}, \vec{p}^{--}\right)$are different. From (5) we shall calculate the source terms of the energy and momentum. The antysymmetry of $C\left(f \mid \vec{p}, \vec{p}_{0}, \vec{p}, \vec{p},-\right)$ at transposition $\vec{p} \vec{f} \vec{p}-$ can be exploited to obtain

$$
\begin{aligned}
& \int I_{2}(f, g) d^{3} p=0, \\
& \int \vec{p} I_{2}(f, g) d^{3} p=-\delta(\vec{x}-\vec{u} t) \vec{I}_{2}(f), \\
& \int \varepsilon I_{2}(f, g) d^{3} p=-\delta(\vec{x}-\vec{u} t) I_{2}(f)
\end{aligned}
$$

where

$$
\begin{aligned}
& \vec{I}_{2}(f)=\frac{1}{\pi} \int(\vec{p}-\vec{p}) \frac{d \sigma(s)}{d t} \cdot f v_{r e 1}^{2} \delta^{4}\left(p+p_{0}-p^{\prime}-p^{\prime}\right) d^{3} p^{3} p^{\prime} d^{3} p^{\prime}, \\
& I_{2}(f)=\frac{1}{\pi} \int\left(\varepsilon-\varepsilon^{\prime}\right) \frac{d \sigma(s)}{d t} \cdot f v_{r e 1}^{2} \delta^{4}\left(p+p_{0}-p^{\prime}-p^{\prime}-\right) d^{3} p d^{3} p^{\prime} d^{3} p^{\prime} \cdots
\end{aligned}
$$

Notice, that $\vec{I}_{2}$ is the vector invariant of the Galilei transformation. If we assume $f$ to be the local equilibrium distribution function:

$$
f=f_{0} \equiv n\left(\frac{m}{2 \pi k T}\right)^{3 / 2} \exp \left(-\frac{m}{2 k T}(\vec{v}-\vec{v})^{2}\right)
$$

where

$$
\begin{aligned}
& \mathrm{n} \text { - normalization density } \\
& \mathrm{T} \text { - temperature, } \\
& \overrightarrow{\mathrm{V}} \text { - hydrodynamic velocity field, }
\end{aligned}
$$

then we have only two vectors $\vec{v}$ and $\vec{u}$ to combine. Therefore we can write

$$
I_{2}(f)=m \frac{\vec{V}-\vec{u}}{\tau}
$$


where $\tau$ is the time parameter characteristic for the momentum transfer particle-medium. Under Galilei transformation $\vec{x} \rightarrow \vec{x}^{\prime}=\vec{x}+\vec{v}_{0} t I_{2}(f)$ transforms in the following way

$$
I_{2}(f) \rightarrow I_{2}^{\prime}(f)=I_{2}(f)+\vec{V}_{0} \vec{I}_{2}(f)
$$

it means that in the local equilibrium case

$$
I_{2}\left(f_{0}\right)=m \frac{\left(\vec{V}^{2}-\vec{u}^{2}\right)}{2 \tau}
$$

Now, we can write the hydrodynamic equations describing the nuclear matter penetrated by the particle. We have

$$
\begin{aligned}
& \frac{\partial \rho}{\partial t}+\operatorname{div} \rho \vec{V}=0, \\
& \rho \frac{\partial \vec{V}}{\partial t}+\rho(\vec{V} \vec{V}) \vec{V}=-\nabla P-\frac{m(\vec{V}-\vec{u})}{\tau} \delta(\vec{x}-\vec{u} t), \\
& \frac{\partial}{\partial t}\left(\frac{\rho \vec{V}^{2}}{2}+\rho \varepsilon\right)=-\vec{v} \cdot\left(\rho \vec{V}\left(\frac{\vec{V}^{2}}{2}+h\right)\right)-m\left(\frac{\vec{V}^{2}-\vec{u}^{2}}{2 \tau}\right) \delta(x-\vec{u} t),
\end{aligned}
$$

where

$$
\begin{aligned}
& \rho \text { - density, } \\
& P \text { - pressure, } \\
& \varepsilon \text { - energy } \\
& h \text { - enthalpy }
\end{aligned}
$$

of the mass unit.

In (14) and (15) there appeared source terms owing to the energy and momentum transfer from the particle to the medium. The parameter $\tau$ can be interpreted as a relaxation time. (A similar idea have been applied to the problem of particle-nuclear matter interaction in the high energy case [5]).

We can also check that the system of equations (13-(15) is not entropy conserving. After simple calculations we can find

$$
\frac{\mathrm{dS}}{\mathrm{dt}}=\frac{1}{\rho \mathrm{T}} \frac{\mathrm{m}(\overrightarrow{\mathrm{V}}-\overrightarrow{\mathrm{u}})^{2}}{2 \tau} \delta(\overrightarrow{\mathrm{x}}-\overrightarrow{\mathrm{u} t}) \neq 0
$$


where $S$ is the entropy of the mass unit.

At the end we can obtain a crude estimation of the $\tau$ parameter. In the incident particle coordinate system $v_{r e 1}=\frac{p}{m}$ and

$$
\frac{m}{\tau}=\frac{1}{\vec{V}^{2}} \int d^{3} p \vec{V} f^{(o)}(p) \frac{p^{2}}{m^{2}} \int d^{3} p-(\vec{p}-\vec{p} \cdot) \frac{d \sigma(s)}{d t} \int d^{3} p--\delta^{4}(p-p-p-\cdots)
$$

We can integrate over $\mathrm{d}^{3} \mathrm{p}^{\cdots}$. In this special coordinate system $\mathrm{s}=2 \mathrm{ME}, \mathrm{dt}=\frac{1}{\pi}\left(\mathrm{pP}^{-}\right) \mathrm{d} \Omega$ where $\mathrm{d} \Omega$ is a spherical volume element. Therefore

$$
\frac{m}{\tau}=\frac{1}{m V} \int d^{3} p \overrightarrow{V f}^{(o)}(p) \int d^{3} p^{-}\left(\vec{p}-\vec{p}^{-}\right) \frac{1}{\cos \theta}\left(\frac{d \sigma}{d \Omega}\right) \delta\left(p^{\prime} \cos \theta-p^{-2}\right) .
$$

The result of the integration over $\mathrm{d}^{3} \mathrm{p}^{\prime}$ is a vector. But we have only one "free" vector $\overrightarrow{\mathrm{p}}$. It means that we can write

$$
\int d^{3} p^{\prime}\left(\vec{p}-\vec{p}^{\prime}\right) \frac{1}{\cos \theta}\left(\frac{d \sigma}{d \Omega}\right) \delta\left(p p^{\prime} \cos \theta-p^{-2}\right)=a \vec{p},
$$

where

$$
\begin{aligned}
& a=p \tilde{\sigma}(\mathrm{p}) \\
& \tilde{\sigma}(\mathrm{p})=\int\left(1-\cos ^{2} \theta\right)\left(\frac{\mathrm{d} \sigma}{\mathrm{d} \Omega}\right) \mathrm{d} \Omega .
\end{aligned}
$$

Finally we have

$$
\frac{m}{\tau}=\int \frac{\vec{V} \vec{p}}{\overrightarrow{m V}^{2}} \quad f^{o}(p) p \tilde{\alpha}(p) d^{3} p
$$

We assume now that the nucleon-nucleon cross section is isotropic and we substitute the Mitropolis approximate value for $\sigma$ given in [6]. We put $u$ to be of the order of the sound velocity in the nuclear matter. The resulting value for $\tau: 4 \cdot 10^{-24} \mathrm{C}$. It is obvious that quantum corrections can change essentially this value.

\section{REFERENCES}

[1] V.M.Kolomietz, H.H.K.Tang, Phys.Sc. 24, 915(1981), 
R.W.Hasse, Proc. of the Topical Meeting on Nuclear Fluid Dynamics, Trieste (1982), and other papers in this volume,

[2] G.Eckart, G.Holzwarth, Proc.of the Topical Meeting on Nuclear Fluid Dynamics, Trieste (1982) and references in this paper,

[3] A.A.Amsden, F.H.Harlow, J.R. Nix, Relativistic Nuclear Fluid Dynamics P.R. C15, 2059(1977),

V.M.Galitskii, J.N.Mishustin, Yad.Fiz.29, 363(1979)

[4] H.S.Köhler, N.P. A378, 181(1982)

[5] V.A.Khangulian, V.A.Galitzky, Ju.B.Ivanov, ZETF Lett. 33, 484(1981)

[6] B.S,Barashenkov, V.D.Toneev, High energy particle interaction with nucleus (1973) (in Russian) 